\title{
The Optical Spectral Slope Variability for 17 Palomar-Green QSOs
}

\author{
X. Pu, W. Bian \& K. Huang \\ Department of Physics and Institute of Theoretical Physics, Nanjing Normal University, \\ Nanjing 210097, China \\ email: whbian@njnu.edu.cn
}

Variability phenomenon in active galactic nuclei (AGNs) provides a powerful constrain on their central engine. For a long time the long-term multi-wavelength photometric monitoring plays an important role in obtaining the optical variability of QSOs. However, it just monitors the flux variability in a few points in the wavelength which usually consists of many components including continuum and strong emission line (although the photometric data are accurate). So it is necessary to study the optical spectral variability using spectroscopic monitoring data.

Up to now, the AGNs spectral variability has focused on about 30 individual objects for a long-term of decades. Kaspi et al. (2000) presented results from a spectrophotometrically monitoring program of a well-defined sample of 28 PG QSOs. Good time sampling $(\geqslant 20)$ and 7.5-years long-term monitoring make these QSOs the best objects in the studying of the spectral slope variability. We use the power law formulae, $f_{\nu} \propto \nu^{-\alpha}$ $\left(f_{\lambda} \propto \lambda^{-2+\alpha}\right)$, to approximately fit the optical continuum spectrum of AGNs. Here we mainly study the relation between $\alpha$ and continuum flux $\log f_{5100}$. The main conclusions can be summarized as follows (for more detail, please refer to $\mathrm{Pu}$ et al. 2006):

- Using the continuum bands suggested by Kaspi et al. (2000), we found that, in 7.5-years long-term observation, all 17 PG QSOs showed obvious optical spectral slope variability. Most of these 17 PG QSOs showed anti-correlation between the spectral slope $\alpha$ and the rest-frame $5100 \AA$ continuum flux $\log f_{5100}$ while five of them showed strong anti-correlation between $\alpha$ and $\log f_{5100}(R>0.5)$.

- For the ensemble of these 17 PG QSOs, a strong anti-correlation $(R=-0.57)$ between the average spectral slope $\alpha$ and the average rest-frame $5100 \AA$ luminosity $\log L_{5100}$ is found while a median anti-correlation $(R=-0.27)$ is found between spectral slope changes $\delta \alpha$ and continuum flux variations $\delta \log f_{5100}$ indicating a hardening of the spectrum during bright phases.

- The disk instability model can qualitatively explain the anti-correlation between the spectral slope $\alpha$ and the continuum flux $\log f_{5100}$ for individual QSOs. However, the global variance of the accretion rates would not necessarily account for the global slope variance for the ensemble of our PG QSOs sample. Accretion disk (jet) instability models with other mechanisms associated with changes in the accretion processes are promising.

This work has been supported by the NSFC (Nos. 10403005, 10473005, 10325313, 10233030 and 10521001) and the Science-Technology Key Foundation from Education Department of China (No. 206053).

\section{References}

$\mathrm{Pu}$ X., Bian W., \& Huang K. 2006, MNRAS, in press.

Kaspi S., Smith P. S., Netzer H. et al. 2000, ApJ 533, 631. 